\section{Cureus}

Received 12/31/2017

Review began 01/17/2018

Review ended 01/19/2018

Published 01/23/2018

\section{(c) Copyright 2018}

Baig et al. This is an open access article distributed under the terms of the Creative Commons Attribution License CC-BY 3.0., which permits unrestricted use, distribution, and reproduction in any medium, provided the original author and source are credited.

\title{
Hip Fracture in a Patient with Primary Hyperparathyroidism: Medical and Surgical Lessons
}

\author{
MN Baig ${ }^{1}$, Cathal Mac Dhaibheid ${ }^{2}$, Fintan J. Shannon ${ }^{3}$ \\ 1. Orthopaedics, University Hospital Galway, Galway, IRL 2. Trauma \& Orthopaedics, Galway University \\ Hospital 3. Trauma \& Orthopaedics, Galway University Hospital , Galway, IRL
}

$\square$ Corresponding author: MN Baig, nouman142@gmail.com

Disclosures can be found in Additional Information at the end of the article

\section{Abstract}

Hyperparathyroidism is a condition which can be primary, secondary or tertiary and is characterized by increased calcium levels, low phosphate levels, and elevated parathyroid hormone (PTH) levels. Primary hyperthyroidism can cause severe bone resorption leading to bone pains and pathological fracture. We present the case of a patient with severe primary hyperparathyroidism with an atraumatic fracture at the neck of the femur and multiple medical comorbidities presenting a surgical challenge. While primary hyperparathyroidism is rare, it should be considered during differential diagnoses.

Categories: Endocrinology/Diabetes/Metabolism, Orthopedics

Keywords: hyperparathyroidism, osteitis fibrosa cystica

\section{Introduction}

Parathyroid hormone (PTH) is released by the parathyroid glands located behind the thyroid gland. It is the main hormone involved in calcium hemostasis [1].PTH increases plasma calcium levels by liberating calcium from the bone, increasing intestinal absorption of calcium, and reducing urinary excretion of calcium. PTH also decreases phosphate levels via inhibiting reabsorption in the kidneys.

Hyperparathyroidism is the overproduction of PTH, a condition that may be due to primary, secondary or tertiary causes. Primary hyperparathyroidism is caused by hypersecretion of PTH by parathyroid hyperplasia or adenoma. Biochemically, hyperparathyroidism raises PTH levels and serum calcium levels and decreases serum phosphate levels. It is usually diagnosed as an incidental finding on routine biochemical tests. Clinically, most primary hyperparathyroidism patients are asymptomatic, but some nonspecific symptoms secondary to high calcium levels are notable; these include fatigue, joint aches, weakness, mild depression, and difficulty concentrating [2].

To diagnose primary hyperparathyroidism, we must rule out other causes of parathyroidism and correlate the condition with biochemical results. Most cases are reported in women and peak in the seventh decade of life [3]. Severe bone disease with severe primary hyperparathyroidism is called osteitis fibrosa. Very rarely they present with hip fractures, and it is a challenge to optimize them medically and treat them surgically [4]. We present the case of a 77-year-old woman presenting with hip fracture and a history of primary parathyroidism. 


\section{Cureus}

\section{Case Presentation}

A 77-year-old woman was referred to the emergency department by her general physician with concerns of a three-day history of progressively worsening right hip pain and an inability to fully bear weight on the affected side. The patient reported no history of trauma, fall, or any other injury. Of note, the patient suffered a previous fracture of the left patella the previous year. Her past medical history was significant for progressive Stage V chronic kidney disease; hyperparathyroidism (diagnosed in 2003 and previous subtotal parathyroidectomy was in 2004), and thrombotic microangiopathy.

On examination, we noted no swelling, ecchymosis, or deformity in her right hip. There was no external shortening or rotation of the leg. The main finding on physical examination was her inability to bear weight and a restricted range of movement in her right hip.

Her estimated glomerular filtration rate was $7 \mathrm{~mL} /$ minute, her PTH level was a remarkable $3842.0 \mathrm{ng} / \mathrm{L}$, and her serum calcium was $2.8 \mathrm{mmol} / \mathrm{L}$. All these levels were much higher than the reference ranges.

An x-ray of the anteroposterior pelvis revealed abnormal bone texture in the right proximal femur with a cortical breach superiorly in the femoral neck (Figure 1). Chronic erosive symphysitis of the pubic symphysis had deteriorated relative to the previous $\mathrm{x}$-rays.

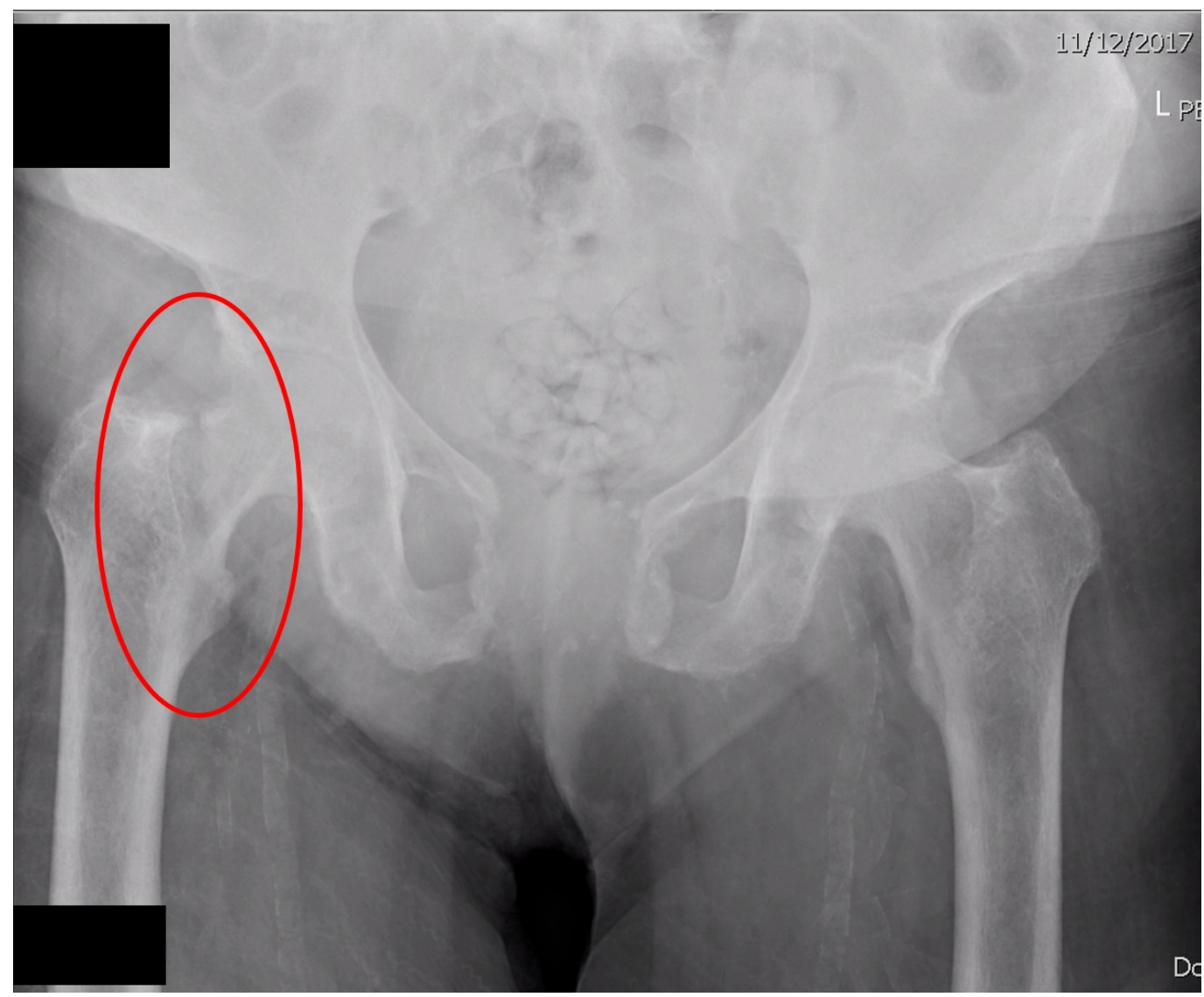

FIGURE 1: X-ray of the pelvis - basicervical fracture

X-ray of the antero-posterior pelvis revealing abnormal bone texture in the right proximal femur with a cortical breach superiorly in the femoral neck. 


\section{Cureus}

The x-rays were followed by a computed tomography (CT) scan of the pelvis to provide further information for an optimal management plan. In the CT scan, the fracture is visible with extensive bone resorption not only in the right femur neck but also in the ischium and contralateral femur (Figure 2).

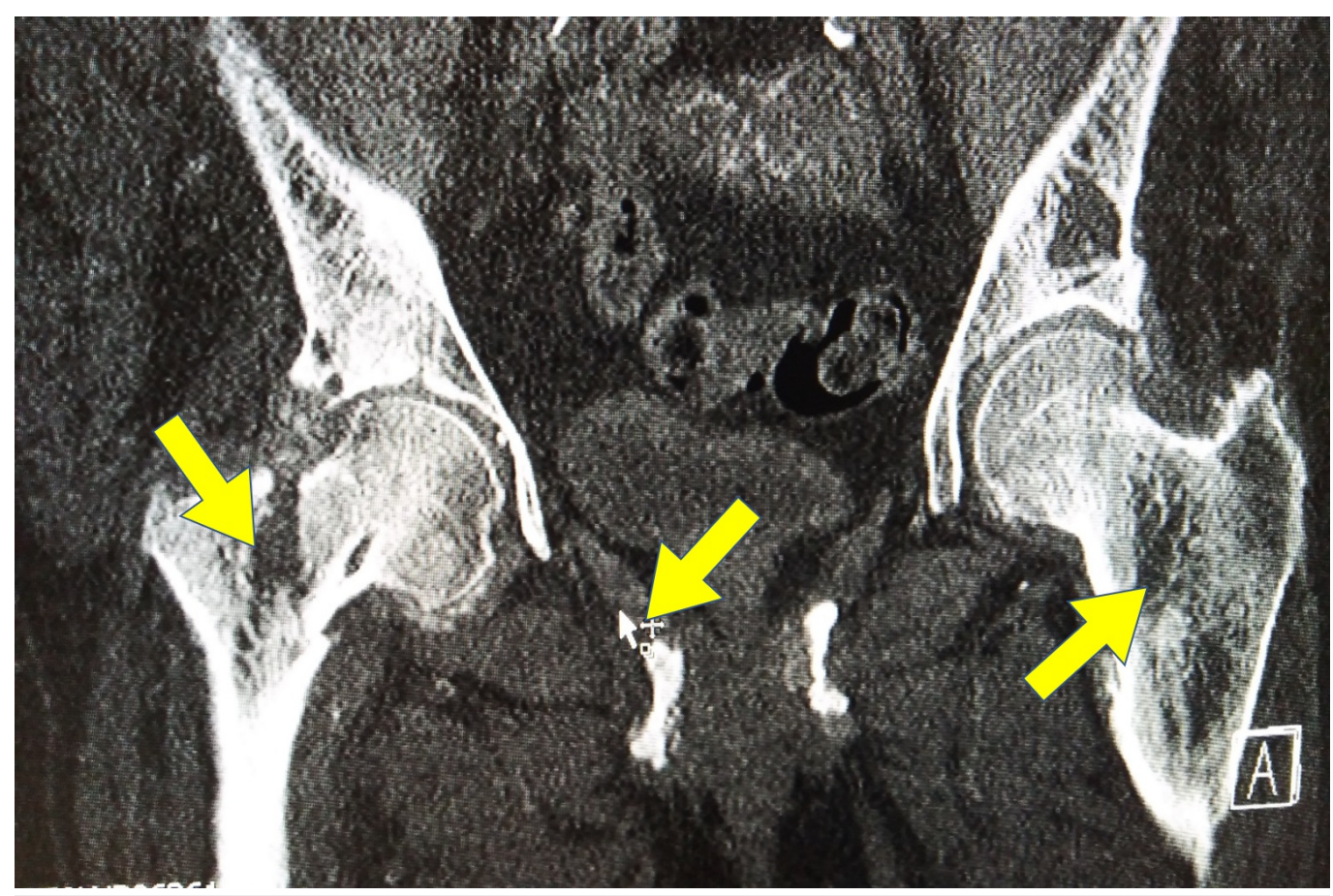

\section{FIGURE 2: Computed tomography (CT) scan of the pelvis -} axial view

The fracture is visible with extensive bone resorption not only in the right femur neck but also in the ischium and contralateral femur.

Chronic symphysitis (visible in Figure 3 and Figure 4 ) was present. It was also present on a previous $\mathrm{x}$-ray three years ago. The extent of chronic symphysitis seen here is rare. 


\section{Cureus}

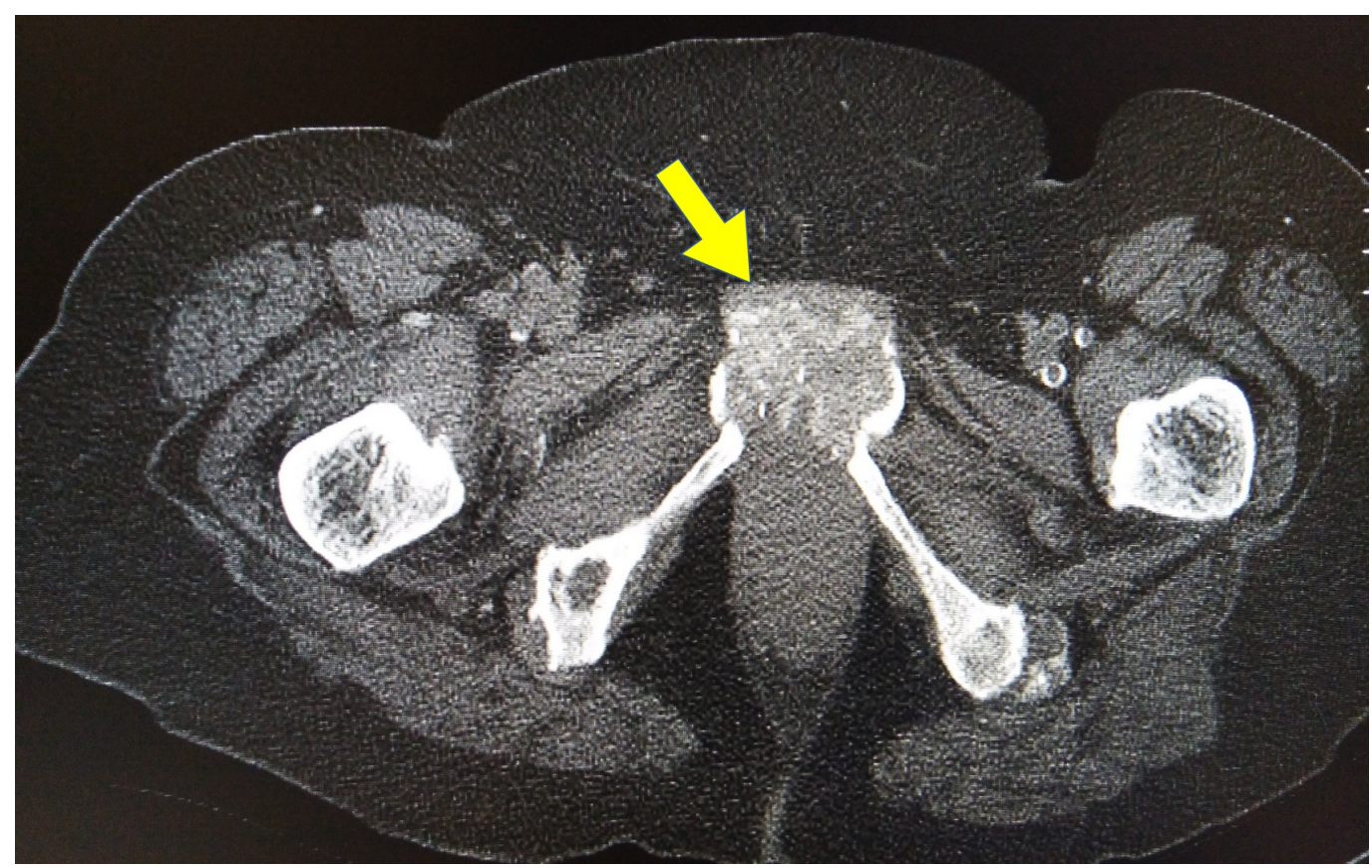

FIGURE 3: Computed tomography (CT) scan of the pelvis coronal view

Chronic symphysitis of pubic symphysis.

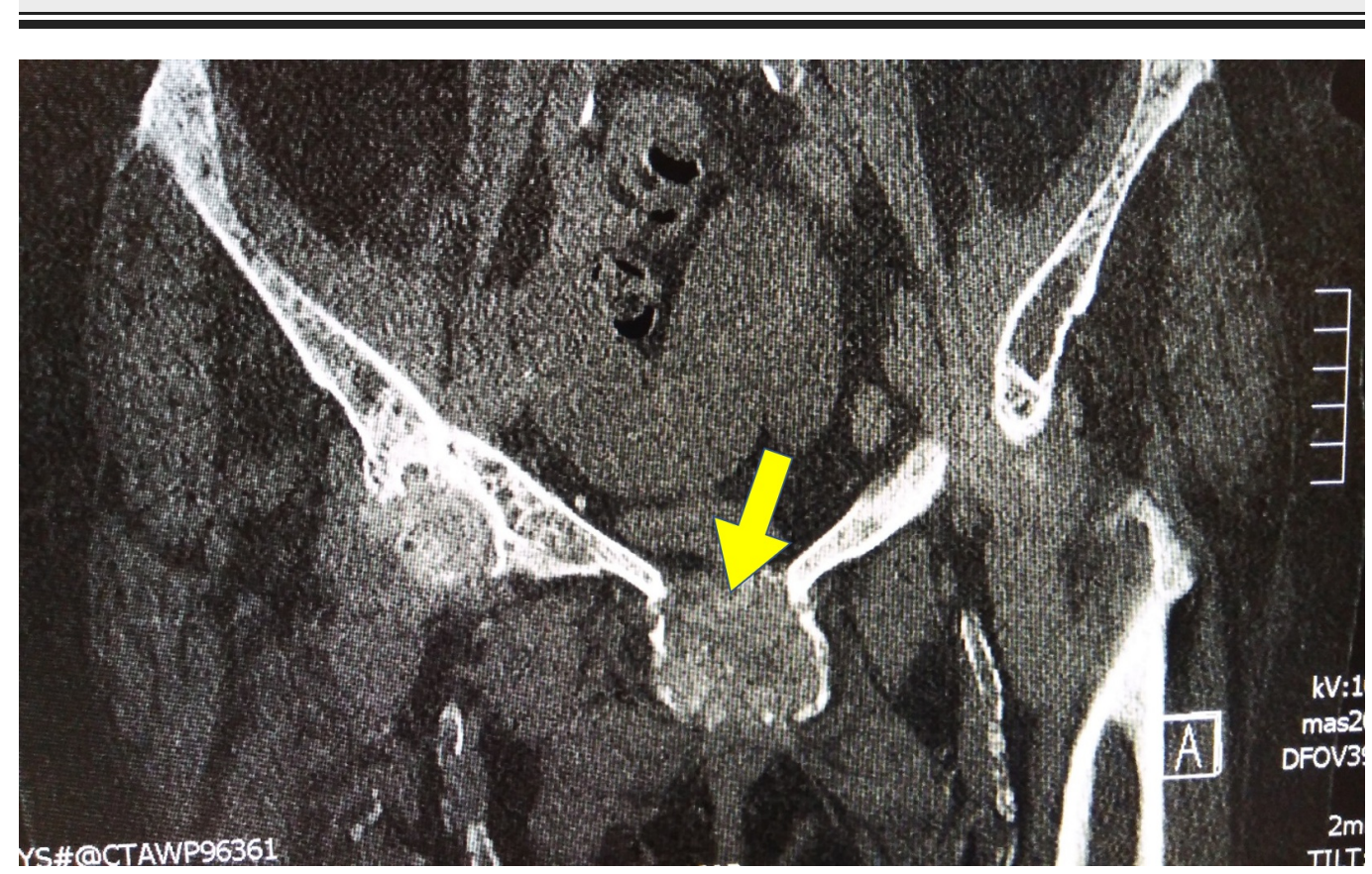

FIGURE 4: Computed tomography (CT) scan of the pelvis axial view

Chronic pubic symphysitis in the axial view.

Her medical conditions presented a challenging surgical situation. Endocrinology, renal, and 
anaesthetic teams were involved to optimize outcomes and to follow the usual practice in our hospital requiring hip fractures operated on within 48 hours of presentation.

She was optimized medically to the anesthetic team's satisfaction to proceed to surgery. Determining the type of operative procedure presented another challenge. As noted on the xray and CT scans, the basicervical neck of the femur fracture is very close to the femoral calcar. The usual surgical procedure in these cases is to insert a dynamic hip screw or short cephalomedullary nail. However, given the quality of the bone tissue and the extent of bone resorption, we decided against these procedures and chose a bipolar hemiarthroplasty.

She recovered from anesthetics and slowly started her physiotherapy and rehabilitation. She was also evaluated by the endocrinology and renal teams to treat her underlying medical conditions.

\section{Discussion}

Primary hyperparathyroidism signs and symptoms are summarized in a colloquial rhyme as "stones, bones, abdominal groans, and psychiatric overtones" [5].The stones in this phrase refer to renal stones; the abdominal groans refer to indigestion, nausea, vomiting, and peptic ulcers. The psychiatric overtones refer to depression, lethargy, and loss of memory. The bones refer to osteitis fibrosa cystica (OFC), osteomalacia, and, rarely, Brown tumor of long bones [6].

The above-mentioned phrase was used in the early 1970s because, at that time, primary hyperparathyroidism was diagnosed in its advanced stages. Today, especially in western countries, this condition is incidentally diagnosed at very early stages thanks to routine biochemical investigations [7-8]. However, rare cases of advanced disease still present where bone structure and other conditions match the patient case presented.

OFC is characterized by bone pains, pathological fractures, and skeletal deformities [7]. Brown tumor is a unifocal or multifocal condition with increased osteoclastic activity, bone resorption, demineralization, and replacement with loose connective tissue [7].

PTH indirectly stimulates osteoclasts by binding to receptors on osteoblasts, inducing RANK-L (receptor activator of nuclear factor kappa $\beta$ ligand) and M-CSF (macrophage colonystimulating factor), causing the overstimulation of bone resorption. RANK-L is an important factor for signaling of transcription factors which lead to osteoclast differentiation. M-CSF stimulates the expression of RANK-L [9].

\section{Conclusions}

While advanced symptomatic primary hyperparathyroidism bone disease is rare, it should be considered in the differential diagnosis especially given the biochemical changes in serum calcium and PTH levels.The patients presenting with hyperparathyroidism and skeletal fractures should be treated not only surgically but also be evaluated medically.

\section{Additional Information Disclosures}

Human subjects: Consent was obtained by all participants in this study. Conflicts of interest: In compliance with the ICMJE uniform disclosure form, all authors declare the following: Payment/services info: All authors have declared that no financial support was received from any organization for the submitted work. Financial relationships: All authors have declared that they have no financial relationships at present or within the previous three years with any 
organizations that might have an interest in the submitted work. Other relationships: All authors have declared that there are no other relationships or activities that could appear to have influenced the submitted work.

\section{References}

1. Bilezikian JP, Silverberg SJ: Asymptomatic primary hyperparathyroidism. N Engl J Med. 2004, 350:1746-51. 10.1056/NEJMcp032200

2. Eller-Vainicher C, Filopanti M, Palmieri S, et al.: Bone quality, as measured by trabecular bone score, in patients with primary hyperparathyroidism. Eur J Endocrinol. 2013, 1:155-62. 10.1530/EJE-13-0305

3. Marcocci C, Cetani F: Primary hyperparathyroidism. N Engl J Med. 2011, 365:2389-97. 10.1056/NEJMcp1106636

4. Baig MN, Curtin W, Callaghan MA, Murphy CG: Unexpected outcome (positive or negative) including adverse drug reactions: catastrophic cement reaction following cementation for megaprosthesis for proximal femoral fracture. BMJ Case Reports. 2017, 23-2017. 10.1136/bcr2017-220158

5. Turner JJO: Hypercalcaemia and primary hyperparathyroidism. Medicine. 2017, 45:551-4. 10.1016/j.mpmed.2017.06.010

6. Misiorowski W, Czajka-Oraniec I, Kochman M, Zgliczyński W, Bilezikian JP: Osteitis fibrosa cystica-a forgotten radiological feature of primary hyperparathyroidism. Endocrine. 2017, 58:380-5. 10.1007/s12020-017-1414-2

7. Lewiecki EM, Miller PD: Skeletal effects of primary hyperparathyroidism: bone mineral density and fracture risk. J Clin Densitom. 2013, 16:28-32. 10.1016/j.jocd.2012.11.013

8. Baig MN, Curtin W: A simple and easy intramedullary lavage method to prevent embolism during and after reamed long bone nailing. Cureus. 2017, 9:e1609. 10.7759/cureus.1609

9. Bassett JHD, Williams GR: Role of thyroid hormones in skeletal development and bone maintenance. Endocr Rev. 2016, 37:135-187. 10.1210/er.2015-1106 